\title{
Tecnologias voltadas para a hipertensão arterial sistêmica: análise documental da produção de conhecimento no Brasil
}

Technologies for systemic arterial hypertension: documentary analysis of knowledge production in Brazil

Tecnologías orientadas a la hipertensión arterial sistémica: análisis documental de la producción de conocimiento en Brasil

Recebido: 31/12/2020 | Revisado: 02/01/2021 | Aceito: 05/01/2021 | Publicado: 07/01/2021

Gabriela Oliveira

ORCID: https://orcid.org/0000-0002-9008-6201 Universidade Federal de Santa Maria, Brasil E-mail: gabrielabockenf@gmail.com

Maria Denise Schimith

ORCID: https://orcid.org/0000-0002-4867-4990 Universidade Federal de Santa Maria, Brasil E-mail: ma.denise2011@gmail.com

Cândida Caniçali Primo

ORCID: https://orcid.org/0000-0001-5141-2898 Universidade Federal do Espírito Santo, Brasil E-mail: candidaprimo@gmail.com

Vanessa de Arruda Marafiga

ORCID: https://orcid.org/0000-0002-2721-4370 Universidade Federal de Santa Maria, Brasil E-mail: vanessaamarafiga@gmail.com

Gabriel da Silva Puhl

ORCID: https://orcid.org/0000-0002-5933-8159 Universidade Federal de Santa Maria, Brasil E-mail: gabrielspuhl@gmail.com

Daiana Cristina Wickert

ORCID: https://orcid.org/0000-0001-7180-1428 Universidade Federal de Santa Maria, Brasil E-mail: daianacristinaw@gmail.com

\begin{abstract}
Resumo
Objetivo: analisar o conhecimento construído em teses e dissertações da Pós-Graduação brasileira acerca das tecnologias para a hipertensão arterial sistêmica. Método: pesquisa documental, realizada em julho 2020, no Catálogo de Teses e Dissertação da Coordenação de Aperfeiçoamento de Pessoal de Nível Superior, mediante duas estratégias de busca: tecnologia AND hipertens* e tecnologia AND "pressão arterial". Após aplicar os critérios de seleção, 21 produções compuseram o corpus final para análise. Resultados: a maioria das produções eram dissertações, produzidas na região Nordeste, pela área da enfermagem, no ano de 2018 e com o método de pesquisa metodológica. Predominaram tecnologias educacionais, com o propósito de educação em saúde, direcionada à prevenção e controle da hipertensão arterial sistêmica. Ainda, foram desenvolvidas tecnologias para o ensino e aperfeiçoamento da medida da pressão arterial, de educação permanente em saúde e, para o gerenciamento do atendimento aos usuários com hipertensão arterial sistêmica. Considerações Finais: as tecnologias em saúde contribuem para a assistência prestada aos usuários com hipertensão arterial sistêmica. Tecnologias para a educação em saúde são tendências da produção do conhecimento, constituindo-se como potentes estratégias. Infere-se a importância de desenvolver também tecnologias voltadas à educação permanente em saúde e para a gestão do cuidado.
\end{abstract}

Palavras-chave: Hipertensão; Tecnologia; Saúde.

\begin{abstract}
Objective: to analyze the knowledge built in theses and dissertations of the Brazilian Graduation Degree about the technologies for systemic arterial hypertension. Method: documentary research, carried out in July 2020, in the Thesis and Dissertation Catalog of the Coordination for the Improvement of Higher Education Personnel, through two search strategies: technology AND hypertens* and technology AND "blood pressure". After applying the selection criteria, 21 productions composed the final corpus for analysis. Results: most of the productions were dissertations, produced in the Northeast region, by the nursing area, in 2018 and with the methodological research method. Educational technologies were predominant, with the purpose of health education, focused on the prevention and control of systemic
\end{abstract}


arterial hypertension. Moreover, technologies for teaching and improving blood pressure measurement, providing continuing health education and managing the care of users with systemic arterial hypertension were also developed. Final Considerations: health technologies contribute to the care provided to users with systemic arterial hypertension. Technologies for health education are trends of the production of knowledge, constituting themselves as powerful strategies. It is inferred that the development of technologies for continuing health education and for care management is also important.

Keywords: Hypertension; Technology; Health.

\section{Resumen}

Objetivo: analizar el conocimiento construido en las tesis y disertaciones del Posgrado Brasileño acerca de las tecnologías para la hipertensión arterial sistémica. Método: investigación documental, realizada en julio de 2020, en el Catálogo de Tesis y Disertaciones de la Coordinación de Perfeccionamiento de Personal de Educación Superior, utilizando dos estrategias de búsqueda: tecnología AND hipertens* y tecnología AND "presión arterial". Después de aplicar los criterios de selección, 21 producciones compusieron el corpus final de análisis. Resultados: la mayoría de las producciones eran disertaciones, producidas en la región Nordeste, por el área de la enfermería, en el año 2018 y empleando el método de investigación metodológica. Hubo predominio de las tecnologías educativas, con el propósito de la educación en salud, orientadas a la prevención y al control de la hipertensión arterial sistémica. Además, se desarrollaron tecnologías para enseñar y mejorar la medición de la presión arterial, la educación continua en salud y la gestión de la atención a usuarios con hipertensión arterial sistémica. Consideraciones finales: las tecnologías de salud contribuyen a la asistencia prestada a usuarios con hipertensión arterial sistémica. Las tecnologías para la educación en salud son tendencias de la producción de conocimiento, constituyéndose como potentes estrategias. Se deduce la importancia de desarrollar también tecnologías dirigidas a la educación continua en salud y para la gestión de la atención.

Palabras clave: Hipertensión; Tecnología; Salud.

\section{Introdução}

A hipertensão arterial sistêmica (HAS) é uma condição crônica multifatorial, caracterizada por níveis pressóricos elevados e sustentados e está fortemente associada aos hábitos de vida dos indivíduos (Sociedade Brasileira de Cardiologia-SBC, 2016). Se não tratada e controlada potencializa o desencadeamento de doenças cardiovasculares, cerebrovasculares e renais, além de gerar altos custos econômicos e sociais (Pinto \& Rodrigues, 2018).

Dessa forma, é importante considerar a severidade da HAS não controlada, como um dos principais fatores para o infarto agudo do miocárdio (IAM), acidente vascular cerebral (AVC), insuficiência cardíaca (IC), nefropatias, retinopatias e diabetes mellitus. Sendo assim, quanto mais precocemente for diagnosticada, tratada de forma adequada e com os cuidados realizados continuamente, maiores serão as probabilidades de se evitar complicações e elevados custos sociais e econômicos (SBC, 2016).

Quanto à abordagem terapêutica da HAS, ela se baseia em tratamento medicamentoso e não medicamentoso. Dessa maneira, a atenção à pessoa com HAS precisa ser realizada de forma conjunta, entre usuário, familiares/cuidadores e profissionais de saúde, cujas ações implementadas no cuidado impactam de forma positiva na adesão ao tratamento. Nessa perspectiva, o enfermeiro, por meio do conhecimento científico e do seu papel como educador, assume protagonismo na promoção da saúde e prevenção de riscos e agravos dessa doença, uma vez que garante condutas que abrangem desde o esclarecimento de dúvidas acerca da terapia medicamentosa até o incentivo de adaptação e de mudanças nos hábitos de vida (Aguiar et al., 2018).

À vista disso, mudanças no processo de trabalho em saúde e na atenção a pessoa com HAS deve-se pautar na efetivação dos princípios do Sistema Único de Saúde (SUS) e na utilização das tecnologias em saúde adequadas para garantir essa assistência (Engela et al., 2018). A Portaria No 2.510, de 19 de dezembro de 2005, do Ministério da saúde, busca incentivar o desenvolvimento científico e tecnológico e define tecnologias em saúde como: medicamentos, materiais, equipamentos e procedimentos, sistemas organizacionais, educacionais, de informações e de suporte, e programas e protocolos assistenciais, por meio dos quais a atenção e os cuidados com a saúde são prestados à população.

Diante dos resultados positivos que vêm sendo obtidos mediante a percepção da eficácia do atrelamento entre as tecnologias em saúde com as demandas dos indivíduos com doenças crônicas não transmissíveis (DCNT), constata-se que fazse necessário o aprimoramento das tecnologias existentes e a construção de novas na atenção a pessoa com HAS, uma vez que 
se deve estimular a participação dos usuários em atividades educativas, reforçar a importância da terapia medicamentosa e favorecer as mudanças dos hábitos de vida (Costa, Santos \& Ferreira, 2019).

O uso de tecnologias na área da saúde está em ascensão, o que torna relevante compreender como acontece a produção das mesmas, a fim de direcionar futuras pesquisas e a utilização na prática profissional. Diante disso, questiona-se: qual a tendência da produção científica brasileira, na área da saúde, acerca das tecnologias voltadas para a hipertensão arterial sistêmica? Este estudo teve como objetivo analisar o conhecimento construído em teses e dissertações da Pós-Graduação brasileira acerca das tecnologias para a hipertensão arterial sistêmica.

\section{Metodologia}

Trata-se de um estudo documental, do tipo descritivo e exploratório, realizado no mês de julho de 2020, a partir da análise das produções (teses e dissertações) disponíveis no Catálogo de Teses e Dissertações da Coordenação de Aperfeiçoamento de Pessoal de Nível Superior (CAPES), vinculado ao Ministério da Educação brasileiro. Compreende-se por pesquisa documental a análise de documentos primários, que corroboram com o objeto de estudo em questão (Gil, 2010).

A busca ocorreu de forma on-line, mediante duas estratégias: tecnologia AND hipertens* e tecnologia AND "pressão arterial”, ressalta-se que as duas estratégias e a ferramenta do “*” aplicada ao radical da palavra hipertensão, foram utilizadas para abranger o maior número de documentos. Aplicou-se o filtro "Ciências da Saúde" no campo Grande Área Conhecimento, foram considerados critérios de inclusão: estudos que respondem à questão de pesquisa e que trabalharam com tecnologias submetidas a processos de construção, validação e/ou avaliação. Foram excluídas as pesquisas indisponíveis e aquelas desenvolvidas com instrumento/questionário/formulário, as produções duplicadas foram consideradas apenas uma vez.

A busca inicial, utilizando as duas estratégias, resultou em 590 produções, das quais 319 estavam vinculadas à grande área das Ciências da Saúde, dessas, 61 estudos não eram da temática, 218 não responderam à questão norteadora, 11 trabalharam com tecnologias que não foram submetidas a processos de elaboração, validação e/ou avaliação, quatro estudos abordaram instrumento/questionário/formulário, quatro produções encontravam-se indisponíveis e 21 estudos foram incluídos, compondo o corpus final.

A análise dos documentos iniciou pela extração das informações de cada estudo, sendo: título e ano de defesa, Instituição de Ensino Superior (IES), método, tipo de tecnologia, público alvo, descritores utilizados, além dos dados sobrescritos foram analisados os resultados e discussão de cada pesquisa. Acrescenta-se que os dados quantitativos referentes a caracterização dos documentos, foram analisados com frequências absolutas (n) e relativas (\%), além disso, os resultados qualitativos, foram agrupados conforme aproximação e similaridade, favorecendo a organização e apresentação. Os resultados foram discutidos à luz da literatura relacionada ao tema, visando o alcance do objetivo proposto.

Como trata-se de um estudo de análise documental não se faz necessária a aprovação do Comitê de Ética em Pesquisa. Ainda, as informações analisadas nas produções que compuseram o corpus do presente estudo foram apresentadas conforme a produção original, sem alteração.

\section{Resultados}

Apresenta-se na sequencia o Quadro 1, contendo a identificação e os títulos das pesquisas que foram analisadas no presente estudo. 
Quadro 1 - Identificação (ID) e títulos das teses e dissertações brasileiras acerca das Tecnologias direcionadas à Hipertensão Arterial Sistêmica, 2020.

\begin{tabular}{|c|c|}
\hline ID & Título do Estudo \\
\hline E1 & $\begin{array}{l}\text { Medida indireta da pressão arterial: avaliação de programa de educação permanente oferecido em } \\
\text { dispositivo móvel. }\end{array}$ \\
\hline E2 & Concepção de um Sistema para o Gerenciamento de Pacientes Direcionado à Hipertensão Arterial. \\
\hline $\mathbf{E 3}$ & Criação e validação de uma cartilha educativa sobre excesso ponderal para o adulto com hipertensão. \\
\hline $\mathbf{E 4}$ & $\begin{array}{l}\text { Formação Permanente de agentes comunitários de saúde em Hipertensão Arterial utilizando Ambiente } \\
\text { Virtual de Aprendizagem. }\end{array}$ \\
\hline E5 & $\begin{array}{l}\text { O impacto de um manual de orientações na adesão ao tratamento medicamentoso da hipertensão arterial } \\
\text { e qualidade de vida dos idosos. }\end{array}$ \\
\hline E6 & Construção e avaliação de curso online para pessoas cegas sobre prevenção da hipertensão arterial. \\
\hline E7 & $\begin{array}{l}\text { Construção e validação de tecnologia educacional como subsídio à ação do enfermeiro na promoção da } \\
\text { qualidade de vida e adesão ao tratamento de pessoas com hipertensão. }\end{array}$ \\
\hline E8 & Eficácia de estratégia educativa para adesão ao tratamento anti-hipertensivo: Programa MeTTA. \\
\hline E9 & $\begin{array}{l}\text { Proposta de tecnologia educativa na prevenção do risco da hipertensão na gravidez - uma construção } \\
\text { coletiva. }\end{array}$ \\
\hline E10 & Construção e validação de uma tecnologia para o gerenciamento da linha do cuidado ao hipertenso. \\
\hline E11 & $\begin{array}{l}\text { A influência da tecnologia educacional híbrida blended learning na adesão terapêutica e na qualidade } \\
\text { de vida do indivíduo hipertenso. }\end{array}$ \\
\hline $\mathbf{E 1 2}$ & $\begin{array}{l}\text { Consulta de enfermagem - tecnologia educativa em saúde para o autocuidado da pessoa com hipertensão } \\
\text { arterial. }\end{array}$ \\
\hline E13 & $\begin{array}{l}\text { Consulta de enfermagem aos hipertensos da estratégia saúde da família: validação e desenvolvimento } \\
\text { de software. }\end{array}$ \\
\hline E14 & Criação e validação de uma cartilha educativa sobre a síndrome hipertensiva gestacional. \\
\hline E15 & $\begin{array}{l}\text { A influência do ambiente virtual de aprendizado (AVA) na adesão terapêutica e no efeito do jaleco } \\
\text { branco. }\end{array}$ \\
\hline E16 & $\begin{array}{l}\text { Validação da tecnologia educativa em saúde - plano de metas para o engajamento do hipertenso no } \\
\text { autocuidado-PMEAc. }\end{array}$ \\
\hline E17 & $\begin{array}{l}\text { Gerenciamento em Atenção Básica: desenvolvimento e avaliação de software-protótipo para a porta de } \\
\text { entrada da Estratégia Saúde da Família. }\end{array}$ \\
\hline E18 & $\begin{array}{l}\text { Manual educativo online acessível sobre hipertensão arterial: avaliação da aprendizagem de cegos e } \\
\text { videntes. }\end{array}$ \\
\hline E19 & $\begin{array}{l}\text { Elaboração de uma hipermídia educacional para o ensino do procedimento de medida de pressão arterial } \\
\text { para utilização em ambiente digital de aprendizagem. }\end{array}$ \\
\hline E20 & $\begin{array}{l}\text { Uso do web aplicativo como estratégia para o ensino da medida da pressão arterial na perspectiva dos } \\
\text { acadêmicos de enfermagem Alfenas/MG } 2018 \text {. }\end{array}$ \\
\hline $\mathbf{E 2 1}$ & $\begin{array}{l}\text { Cuidado de enfermagem à saúde da gestante: aplicativo móvel para o autocuidado no controle da } \\
\text { pressão arterial. }\end{array}$ \\
\hline
\end{tabular}

Fonte: Resultados da pesquisa (2020). 


\subsection{Caracterização das Teses e Dissertações acerca das Tecnologias direcionadas à Hipertensão Arterial Sistêmica}

Em relação as principais características das produções analisadas, dos 21 (100\%) trabalhos selecionados, sete (33,3\%) identificaram-se como Teses e 14 (66,7\%) como Dissertações, sendo que dessas, três $(21,4 \%)$ eram oriundas de Mestrado Profissional e 11 (78,6\%) de Mestrado Acadêmico.

No total, os trabalhos apresentaram 96 (100\%) descritores, sendo 57 (59,4\%) palavras-chaves em termos diferentes ou em variações e, $17(17,7 \%)$ desses se repetiram ao menos uma vez. Entre os descritores mais frequentes citados nos estudos, o termo hipertensão e suas variações, sendo elas "hipertensão", "hipertensão arterial", "hipertensão gestacional", "hipertensão induzida pela gravidez" e "hipertensão do jaleco branco" apresentaram maiores frequências 14,6\% (n=14). As variações entre "tecnologia educacional", "tecnologia educativa", "tecnologia em saúde", "tecnologia" e "tecnologia da informação", representaram 11,5\% ( $\mathrm{n}=11)$ dos descritores. Já o termo "enfermagem" e sua variação "enfermagem vascular" representaram 10,4\% ( $\mathrm{n}=10)$. Os termos relacionados à educação, também tiveram representatividade no que diz respeito aos descritores, compreendendo "educação em saúde", "educação à distância", "educação permanente em saúde" e "educação" constituindo $7,3 \%(\mathrm{n}=7)$ dos descritores utilizados nos estudos.

A análise da distribuição demográfica dos estudos evidencia que na região Nordeste está concentrada a maior produção sobre a temática, com $57,1 \%(\mathrm{n}=12)$ do total dos estudos, sendo o Estado do Ceará o que concentra o maior número de produções, com 11 documentos (50,0\%). Seguindo, as produções da região Sudeste representaram 38,1\% ( $\mathrm{n}=8$ ) e da região Sul 4,8\% ( $\mathrm{n}=1$ ). No que diz respeito a área do conhecimento conforme a formação do autor, a enfermagem apresentou predomínio de 95,2\% (n=20) e a nutrição de 4,8\% (n=1). Em relação ao ano de publicação e método dos estudos, apresenta-se a seguir a Tabela 1.

Tabela 1 - Caracterização quanto ao ano de publicação e ao método das teses e dissertações brasileiras acerca das Tecnologias direcionadas à Hipertensão Arterial Sistêmica, 2020.

\begin{tabular}{lcc}
\hline ANO DE PUBLICAÇÃO & N & $\%$ \\
\hline 2018 & 05 & 23,8 \\
\hline 2017 & 02 & 9,5 \\
\hline 2016 & 03 & 14,2 \\
\hline 2015 & 05 & 23,8 \\
\hline 2014 & 02 & 9,5 \\
\hline 2013 & 01 & 4,8 \\
\hline 2012 & 01 & 4,8 \\
\hline 2007 & 01 & 4,8 \\
\hline 2006 & 01 & 4,8 \\
\hline MÉTODO & & 38,1 \\
\hline Metodológico & 08 & 19,0 \\
\hline Ensaio Clínico Randomizado e Controlado & 04 & 14,2 \\
\hline Metodológico seguido de Quase Experimental & 03 & 9,5 \\
\hline Pesquisa Aplicada & 02 & 4,8 \\
\hline Descritivo Exploratório & 01 & 4,8 \\
\hline Descritivo Transversal & 01 & 4,8 \\
\hline Exploratório seguido de Metodológico & 01 & 4,8 \\
\hline Pesquisa Avaliativa & 01 & \\
\hline
\end{tabular}

Fonte: Resultados da Pesquisa (2020). 
É possível perceber o predomínio de publicações nos anos de $2018^{(\mathrm{E} 12, \mathrm{E} 16, \mathrm{E} 18, \mathrm{E} 20, \mathrm{E} 21)}$ e $2015^{(\mathrm{E} 4, \mathrm{E} 6, \mathrm{E} 7, \mathrm{E} 13, \mathrm{E} 14)}$, seguido de $2016^{(\mathrm{E} 3, \mathrm{E} 15, \mathrm{E} 17)}, 2017^{(\mathrm{E} 8, \mathrm{E} 11)}, 2014^{(\mathrm{E} 5, \mathrm{E} 9)}$ e os anos de 2013 ${ }^{(\mathrm{E} 10)}, 2012^{(\mathrm{E} 1)}, 2007^{(\mathrm{E} 19)}$ e $2006^{(\mathrm{E} 2)}$ tiveram uma publicação cada. No que concerne ao método, destacaram-se os estudos metodológicos (E6, E9, E10, E12, E13, E16, E20, E21), ensaios clínicos randomizados e $\operatorname{controlados}^{(\mathrm{E} 5, \mathrm{E} 8, \mathrm{E} 11, \mathrm{E} 15)}$, metodológicos seguidos de Quase Experimental ${ }^{(\mathrm{E} 3, \mathrm{E} 7, \mathrm{E} 18)}$, pesquisa aplicada ${ }^{(\mathrm{E} 2, \mathrm{E} 19)}$, e os métodos descritivo exploratório ${ }^{(\mathrm{E} 1)}$, descritivo transversal ${ }^{\mathrm{E} 4)}$, exploratório seguido de metodológico ${ }^{(\mathrm{E} 14)}$ e pesquisa avaliativa ${ }^{(\mathrm{E} 17)}$ com um estudo cada.

\subsection{Distribuição quanto ao tipo, formato e conteúdo das tecnologias analisadas}

Em relação ao tipo de tecnologia a maioria dos autores definiram e/ou trabalharam com tecnologias educacionais (E1, E3. E4, E5, E6, E7, E9, E11, E14, E15, E16, E18, E19, E20, E21). Entre os estudos, a maior parte abordou a educação em saúde (E3, E5, E6, E7, E9, E11, E14, E15,

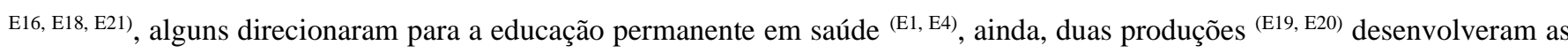
tecnologias voltadas para o ensino na formação profissional. Outras pesquisas abordaram tecnologias voltadas ao gerenciamento (E2, E10, E17). Um estudo definiu como tecnologia móvel ${ }^{(\mathrm{E} 8)}$, e duas produções não especificaram os tipos de tecnologias trabalhadas (E12, E13).

No que diz respeito ao formato, prevaleceram as tecnologias digitais (E1, E2, E4, E6, E8, E10, E11, E13, E15, E17, E18, E19, E20, E21) sendo, aplicativos para dispositivos móveis ${ }^{(\mathrm{E} 1, \mathrm{E} 20, \mathrm{E} 21)}$, softwares (E2, E13, E17), ambientes virtuais para cursos de aprendizado (E4, E6, E11, E15), mensagens de textos (SMS) ${ }^{(\mathrm{E} 8)}$, cartilha eletrônica ${ }^{(\mathrm{E} 10)}$, manual on-line ${ }^{(\mathrm{E} 18)}$, hipermídia ${ }^{(\mathrm{E} 19)}$. Outros estudos analisados, versaram tecnologias impressas (E3, E5, E7, E9, E14) como, cartilhas ${ }^{(E 3, E 5, E 14)}$ e álbum seriado ${ }^{(\mathrm{E} 7}$. Por fim, uma tecnologia foi trabalhada em formato digital e impresso ${ }^{(\mathrm{E} 16)}$ e outra ${ }^{(\mathrm{E} 9)}$ não especificou.

A maioria dos estudos buscou promover a saúde das pessoas com HAS e prevenir agravos, as tecnologias desenvolvidas por essas pesquisas abordaram principalmente os hábitos saudáveis. Nesse sentido, uma pesquisa ${ }^{(\mathrm{E} 3)}$ desenvolveu uma cartilha direcionada a pessoas com excesso ponderal, com o objetivo de favorecer a mudança de hábitos alimentares e a realização de atividades físicas. Outra produção ${ }^{(\mathrm{E} 16)}$ validou uma tecnologia para o engajamento do usuário com HAS para o autocuidado. Ainda, dois estudos trabalharam a adesão ao tratamento e promoção da qualidade de vida dos usuários com HAS, um ${ }^{\text {(E7) }}$ por meio da construção e validação de um álbum seriado e, o outro ${ }^{(\mathrm{E} 5)}$ mediante a aplicação e verificação da eficácia de um manual de orientações. No que concerne aos fármacos anti-hipertensivos, acrescenta-se que um estudo ${ }^{(E 8)}$, testou a eficácia de um programa educativo de mensagens de texto via celular, denominado programa MeTTA, com o propósito de motivar a adesão ao tratamento. Ademais, duas pesquisas (E6, E18) também constituíram tecnologias para prevenção e educação em saúde, todavia, essas produções foram direcionadas à população com deficiência visual.

Dois estudos (E11, E15), que são subprojetos de um protocolo original, assim denominado pelos autores das pesquisas, trabalharam com uma tecnologia educacional na modalidade do Ensino a Distância (EaD) no Ambiente Virtual de Aprendizagem (AVA) intitulada "E-Care da Hipertensão", a qual se tratava de um portal composto por seis módulos que abordavam aspectos gerais da HAS, adesão à terapêutica medicamentosa, nutrição, incentivo a realização de atividades físicas, estresse e a influência sobre a HAS e, por fim, o último módulo constituía-se de um jogo, abordando situações comportamentais para a prevenção e controle da HAS. Os dois estudos ${ }^{\left({ }^{E 11,} \mathrm{E} 15\right)}$ foram direcionados às pessoas com HAS, porém, um ${ }^{(\mathrm{E} 11)}$ teve a finalidade de contribuir com o controle dos níveis pressóricos e a qualidade de vida, por meio da educação em saúde desenvolvida no "E-Care da Hipertensão" e, a outra pesquisa ${ }^{(E 15)}$ trabalhou além da adesão terapêutica e qualidade vida das pessoas com HAS, a hipertensão do avental branco, por meio da mesma tecnologia.

Especificadamente para as gestantes, uma dissertação (E21) elaborou e validou um aplicativo em plataforma móvel (celulares) direcionado para o autocuidado, que constava informações a respeito da HAS, as quais buscavam orientar e instrumentalizar a gestante para a realização do cuidado. Outro estudo ${ }^{(E 14)}$ também direcionou a tecnologia para a educação em 
saúde com as gestantes, propondo contribuir no controle da síndrome hipertensiva. Por fim, uma produção ${ }^{(E 9)}$ teve o propósito de guiar as consultas de pré-natal, contribuindo com a prevenção e o controle do risco de hipertensão durante a gestação.

Outro conteúdo trabalhado nas produções analisadas foi a verificação da pressão arterial, as pesquisas tiveram o propósito de aperfeiçoar e fortalecer a técnica desenvolvida. Duas pesquisas ${ }^{(\mathrm{E} 19 \text {, E20) }}$ tiveram a finalidade, por meio da tecnologia, de ensinar e aprimorar a medida da pressão arterial com estudantes do curso de enfermagem. Outro estudo ${ }^{(E 1)}$ desenvolveu um curso para a equipe de enfermagem (auxiliares, técnicos de enfermagem e enfermeiros) abordando a medida da pressão arterial, o curso construído e aplicado foi fundamentado nos pressupostos da educação permanente em saúde. Corroborando com os pressupostos da educação permanente em saúde, uma dissertação ${ }^{(E 4)}$ trabalhou com um curso de formação permanente com agentes comunitários de saúde, compartilhando conhecimentos e contribuindo com o trabalho desenvolvido por esses profissionais aos usuários com HAS.

Duas produções analisadas abordaram a consulta de enfermagem ${ }^{\text {(E12, E13) }}$. Uma delas ${ }^{(E 12)}$ buscou validar a consulta de enfermagem como tecnologia educativa para o engajamento da pessoa para o autocuidado, dessa forma, o estudo foi fundamentado nos pressupostos teóricos de Dorothea Elizabeth Orem. O outro estudo (E13) trabalhou com a Consulta de Enfermagem desenvolvida a partir da Classificação Internacional para a Prática de Enfermagem (CIPE), buscando contribuir com a padronização e registro da consulta de enfermagem, além de otimizar o tempo para o desenvolvimento.

Além dos conteúdos e projeções acima descritas, algumas pesquisas trabalharam com o enfoque gerencial, sendo que três estudos (E2, E10, E17) utilizaram as tecnologias para a gestão dos serviços de saúde. Um deles ${ }^{(\mathrm{E} 2)}$ desenvolveu um sistema para melhorar o acompanhamento do paciente, permitindo o cadastro, definição de risco, as ações que precisam ser realizadas, além de monitorar a evolução e emitir a fatura dos possíveis custos. Outro ${ }^{(\mathrm{E} 10)}$ produziu uma cartilha eletrônica e propôs $o$ gerenciamento da linha do cuidado aos usuários com HAS contemplando a integralidade das ações e da assistência prestada. E uma pesquisa ${ }^{\left({ }^{(17)}\right)}$ construiu um software - protótipo, para o gerenciamento da agenda programática da Atenção Básica, entre as consultas, destaca-se os usuários com HAS.

No que se refere ao material teórico, as principais referências citadas pelos autores foram os cadernos do Ministérios da Saúde, números 35, 37 e 38, os quais abordam estratégias para o cuidado da pessoa com doença crônica, hipertensão e obesidade, respectivamente. Além desses, as Diretrizes Brasileiras de Obesidade e a VI Diretrizes Brasileiras de Hipertensão.

\section{Discussão}

A área da enfermagem destacou-se na produção dos estudos, os profissionais de enfermagem estão desenvolvendo cada vez mais produções tecnológicas, buscando uma assistência humanizada, integradora e de qualidade. Nesse contexto, considerase as doenças mais prevalentes no cuidado em saúde, como as crônicas não transmissíveis (Rolim, Rabelo, Lobo, Moreira, \& Samelli, 2015).

Autores afirmam que no cuidado direcionado aos usuários com HAS, o profissional enfermeiro, como integrante da equipe multiprofissional, na maioria das vezes, desenvolve o atendimento inicial. A partir disso, é primordial que o enfermeiro realize o acompanhamento do usuário, direcionando o cuidado conforme suas necessidades, buscando a promoção da saúde e a prevenção de agravos e complicações, além de incentivar a corresponsabilização do usuário com o seu cuidado (Mendes, Silva, \& Ferreira, 2018).

A maioria das tecnologias foi desenvolvida com o propósito de educação em saúde, com relação as DCNT, é importante que aconteça o controle efetivo e integral, por meio, principalmente, dos hábitos e estilo de vida saudáveis, nesse sentido a educação em saúde constitui-se como estratégia importante para promover esses cuidados (Salci, Meirelles, \& Silva, 2018). Assim, quanto maior for o conhecimento dos usuários acerca de sua condição, maior será probabilidade para desenvolver as ações para o cuidado adequado. Nesse cenário, destaca-se a corresponsabilização da equipe de saúde, em trabalhar com 
estratégias educacionais para a orientação dos usuários com HAS (Silva, Avelino, Sousa, Valle, \& Figueiredo, 2016). Sendo que estratégias inovadoras favorecem a compreensão e a adesão dos usuários ao tratamento medicamentoso e não medicamentoso (Santana et al., 2020).

As produções analisadas trabalharam com tecnologias que além de informar os usuários a respeito da HAS, buscaram sensibilizar e contribuir com a realização dos cuidados e, consequentemente, com a qualidade de vida. Portanto, as tecnologias podem auxiliar na promoção dos cuidados à saúde, possibilitando melhor compreensão e entendimento, por meio da integração de diversas mídias, linguagens e recursos, permitindo o desenvolvimento de um processo educacional interativo (Pinto, Scopacasa, Bezerra, Pedrosa, \& Pinheiro, 2017).

Uma das atribuições do profissional enfermeiro é a realização da consulta de enfermagem, a qual propicia um momento de diálogo e interação, oportunizando a abordagem singular, além disso, o enfermeiro pode avaliar as necessidades do usuário e identificar as variáveis que interferem positiva ou negativamente na realização do cuidado (Alencar et al., 2017). Dessa forma, as tecnologias que contribuem com a operacionalização da consulta de enfermagem favorecem a assistência ao usuário com HAS.

Algumas tecnologias foram fundamentadas nos pressupostos da educação permanente em saúde, destaca-se a importância em trabalhar com a qualificação dos profissionais, uma vez que, essas estratégias são desenvolvidas a partir das necessidades do cotidiano, abordando diversos temas e construindo conhecimentos (Silva et al., 2016). Embora uma produção tenha realizado um curso de formação para os agentes comunitários de saúde, percebe-se poucas produções, corroborando a esse achado, um estudo de revisão apontou lacunas na abordagem de aspectos teóricos e práticos no processo de trabalho dos agentes comunitários de saúde (Alonso, Béguin, \& Duarte, 2018). Portanto, ressalta-se a importância de pesquisas que desenvolvam estratégias de educação permanente em saúde e, acrescenta-se, que além dos agentes comunitários de saúde, direcionem também aos outros profissionais integrantes da equipe.

Outro aspecto analisado no presente estudo, foram as produções que abordaram a verificação dos níveis pressóricos. A medida da pressão arterial é uma das técnicas mais realizada pela equipe de enfermagem em seu ambiente de trabalho, na maioria das vezes, é considerada simples e de fácil execução, contudo, são necessários cuidados para a obtenção de resultados fidedignos (Oliveira, \& Almeida, 2015). Haja vista que a medida fidedigna é o que vai estabelecer a eficácia no diagnóstico e tratamento, torna-se imprescindível o conhecimento e o domínio por parte dos profissionais e futuros profissionais, acerca do preparo para realização da técnica, os equipamentos adequados e a verificação correta, evidenciando a importância de tecnologias que contribuem para o ensino e o aprimoramento do procedimento realizado.

As tecnologias gerenciais são utilizadas para mediação dos processos de gestão nos serviços de saúde, podendo ser desenvolvidas e aplicadas por profissionais, contribuindo com a organização e a qualidade da assistência prestada (Nietsche, Backes, Colomé, Ceratti, \& Ferraz, 2005). Dessa forma, tecnologias gerenciais direcionadas ao atendimento do usuário com HAS é muito importante, em razão de ter um elevado número de usuários com esta condição, situação que se agrava ao considerar que muitos apresentam dificuldades para o controle, evidenciando a necessidade de organizar o fluxo de atendimento conforme as especificidades de cada caso.

Portanto, as tecnologias podem ser entendidas como resultado de distintos processos a partir da pesquisa e da experiência do cotidiano, os quais possibilitam o desenvolvimento de conhecimentos científicos. Tal processo pode resultar na construção de produtos materiais, ou não, que têm o propósito de propiciar intervenções sobre um tema ou acontecimento prático (Nietsche, Backes, Colomé, Ceratti, \& Ferraz, 2005). Sendo assim, as tecnologias voltadas para a HAS, contribuem com a assistência profissional e o cuidado dos usuários com HAS, favorecendo o controle da condição e a diminuição da morbimortalidade em decorrência dos desfechos cardiovasculares. 


\section{Considerações Finais}

A realização da presente pesquisa possibilitou identificar e analisar a produção científica acerca das tecnologias voltadas para HAS. Embora não tenha sido aplicado nenhum filtro para a área da enfermagem, a mesma foi predominante no desenvolvimento dos estudos, além disso, a maioria tratava-se de dissertações e foi realizada na região Nordeste. Quanto ao delineamento, prevaleceram estudos metodológicos, ensaios clínicos randomizados e controlados, ou ainda, os dois desenvolvidos no mesmo estudo em duas etapas, resultado importante, pois esses métodos vão ao encontro dos processos de construção, validação e/ou avaliação de tecnologias.

A partir da análise documental, conclui-se que a maioria das tecnologias era educacional, envolvendo, principalmente, hábitos saudáveis, com a finalidade de prevenção e controle da HAS, os comportamentos abordados foram alimentação adequada, realização de atividade física, controle dos níveis pressóricos e adesão ao tratamento medicamentoso. Além do estilo de vida saudável, outros estudos, em número reduzido, desenvolveram tecnologias para o aperfeiçoamento da medida da pressão arterial e, para a educação permanente em saúde, ou ainda, tiveram a finalidade de contribuir com o gerenciamento do atendimento aos usuários com HAS.

Considera-se que as tecnologias analisadas são importantes para a prevenção e controle da HAS, por meio do incentivo aos hábitos saudáveis. No entanto, foi possível perceber que há predominância de tecnologias voltadas à educação em saúde, sendo necessário, considerar outros aspectos, como educação permanente em saúde e a gestão do cuidado diante da HAS. Esperase que o presente estudo possa favorecer o desenvolvimento de pesquisas futuras que busquem preencher a lacuna identificada.

\section{Referências}

Aguiar, A. C. T., Santos, Z. M. S. A., Rodrigues, K. A. F., Santos, P. D. S., Júnior, J. I. C. O., \& Rolim, K. M. C. (2018). Repercussão da tecnologia educativa em saúde na adesão de familiares cuidadores no controle da hipertensão arterial ou na prevenção de risco desse agravo. Tecnologias para a promoção e o cuidado em saúde, 50-70. Ed. UECE. https://www.biosanas.com.br/uploads/outros/artigos_cientificos/59/39ab383257a9da8d6421cc9ada1a1490.pdf

Alencar, D. C., Costa, R. S., Alencar, A. M. P. G., Moreira, W. C., Ibiapina, A. R. S, \& Alencar, M. B. (2017). Consulta de enfermagem na perspectiva de usuários com diabetes mellitus na Estratégia Saúde da Família. Revista de Enfermagem UFPE on line, 11(10), 3749-3756. 10.5205/reuol.12834-30982-1SM.1110201707

Alonso, C. M. C., Béguin, P. D., \& Duarte, F. J. C. M. (2018). Trabalho dos agentes comunitários de saúde na Estratégia Saúde da Família: metassíntese. Revista de Saúde Pública, 52(14). https://doi.org/10.11606/s1518-8787.2018052000395

Costa, L. R. L. G., Santos, K. C., \& Ferreira, L. B. (2019). Adesão ao tratamento de hipertensão arterial. Journal of the Health Sciences Institute, 37(4), 351359. https://www3.unip.br/presencial/comunicacao/publicacoes/ics/edicoes/2019/04_out-dez/10V37_n4_2019_p351a359.pdf

Engela, M. H. T., Rodarte, A. C, Júnior, A. R., Seixas, C. T., Viegas, S. M. F., \& Lanza, F. M. (2018). Uso das tecnologias em saúde na atenção básica às pessoas em condições de hipertensão arterial sistêmica. Revista Online de Pesquisa: Cuidado é Fundamental, 10 (1), 75-84. 10.9789/2175-5361.2018.v10i1.75-84

Gil, A. C. (2010). Como elaborar projetos de pesquisa. Atlas.

Mendes, F. A., Silva, M. P., \& Ferreira, C. R. S. (2018). Diagnósticos de enfermagem em portadores de hipertensão arterial na atenção primária. Estação Científica (UNIFAP), 8(1),91- 101. 10.18468/estcien.2018v8n1.p91-101

Nietsche, E. A., Backes, V. M. S., Colomé, C. L. M., Ceratti, R. N., \& Ferraz, F. (2005). Tecnologias educacionais, assistenciais e gerenciais: uma reflexão a partir da concepção dos docentes de enfermagem. Revista Latino-Americana de Enfermagem, 13(3), 344-52. https://www.scielo.br/scielo.php?script=sci_arttext\&pid=S0104-11692005000300009

Oliveira, T. M. F., \& Almeida, T. C. F. (2015). Adequação do manguito durante a medida da pressão arterial: uma revisão integrativa. Ciência \& Saúde, 8 (1), 35-41. https://doi.org/10.15448/1983-652X.2015.1.19419

Pinto, A. C., Scopacasa, L. G., Bezerra, L. L. A. L., Pedrosa, J. V., \& Pinheiro, P. N. C. (2017). Use of information and communication technologies in health education for adolescents: integrative review. Revista de Enfermagem UFPE on line, 11 (2), 634-644. 10.5205/reuol.10263-91568-1-RV.1102201719

Pinto, E. S. O., \& Rodrigues, W. N. (2018) Systematization of Nursing Assistance in Primary Care for people with hypertension. Revista Nursing, 21(237), 2036-40. Obtido de http://www.revistanursing.com.br/revistas/237Fevereiro2018/sistematizacao_da_assistencia_de_enfermagem.pdf

Portaria nº 2.510, de 19 de dezembro de 2005. Institui Comissão para Elaboração da Política de Gestão Tecnológica no âmbito do Sistema Único de Saúde CPGT. http://bvsms.saude.gov.br/bvs/saudelegis/gm/2005/prt2510_19_12_2005.html.

Rolim, L. P., Rabelo, C. M., Lobo, I. F. N., Moreira, R. R., \& Samelli, A. G. (2015). Interação entre diabetes mellitus e hipertensão arterial sobre a audição de idosos. CoDAS. Sociedade Brasileira de Fonoaudiologia, 27 (5), 428-432, 2015. 10.1590/2317-1782/20152014101 
Research, Society and Development, v. 10, n. 1, e19010111624, 2021

(CC BY 4.0) | ISSN 2525-3409 | DOI: http://dx.doi.org/10.33448/rsd-v10i1.11624

Salci, M. A., Meirelles, B. H. S., \& Silva, D. M. G. (2018). Educação em saúde para prevenção das complicações crônicas do diabetes mellitus na atenção primária. Escola Anna Nery - Revista de Enfermagem, 22 (1), 1-6. 10.1590/2177-9465-EAN-2017-0262

Santana, L. C. B., Soares, T. C., Wenzel, A. P. B. P. H., Blanche, B. R., Benevides, L. K. B., Soares T. C., Câmara, G. B., Silva, D. F., \& Souza, E. C. (2020). Promoção à saúde de hipertensos e diabéticos a partir da problematização do território. Research, Society and Development, 9 (1), e141911492. http://dx.doi.org/10.33448/rsd-v9i1.1492

Silva, A. P., Avelino, F. V. S. D, Sousa, C. L. A., Valle, A. R. M. C., \& Figueiredo, M. L. F. (2016). Fatores associados à não adesão ao tratamento da hipertensão arterial sistêmica: uma revisão integrativa. Revista Online de Pesquisa: Cuidado é Fundamental, 8(1), 4047-4055. 10.9789/2175-5361.2016.v8i1.4047-4055

Silva, L. A. A., Pinno, C., Schmidt, S. M. S., Noal, H. C., Gomes, I. E. M., \& Signor, E. (2016). A educação permanente no processo de trabalho de enfermagem. Revista de Enfermagem do Centro Oeste Mineiro, 6(3),2349-2361. 10.19175/recom.v6i3.1027

Sociedade Brasileira de Cardiologia. (2016). $7^{\mathrm{a}}$ Diretriz Brasileira de Hipertensão Arterial. Arq Bras Cardiol., 107 (3), 1-3. http://publicacoes.cardiol.br/2014/diretrizes/2016/05_HIPERTENSAO_ARTERIAL.pdf 